\title{
COMPARAÇÃO DO DESEMPENHO DO CLASSIFICADOR DE NOVIDADES COM O CLASSIFICA- DOR DO VIZINHO MAIS PRÓXIMO NO RECONHECIMENTO FACIAL
}

\author{
Cícero F. F. C. Filho ${ }^{1}$, Thiago A. Falcão $0^{1,2}$, Marly G. F. Costa ${ }^{1}$. \\ 1. Centro de Tecnologia Eletrônica e da Informação, Universidade Federal do Amazonas \\ Av. Rodrigo Otávio, 3000, Coroado, 69077-000 - Manaus, AM, Brasil \\ E-mails: CffCfilholgmail.com, marly.costa@uol.com.br
}

\author{
2. Instituto Nokia de Tecnologia \\ Av. Torquato Tapajós, 7200, Colônia Terra Nova, 69093-415-Manaus,AM, Brasil \\ E-mails: thiagofalcao@gmail.com
}

\begin{abstract}
This paper proposes using the novelty classifier to face recognition. This classifier is based on novelty filters, proposed by Kohonen. The performance of the new classifier is compared with nearest neighbor classifier, using Euclidian distance. The face data base used for this comparison was the ORL. The face data is extracted using PCA and 2DPCA strategies. Some results are presented: recognition rate versus number of auto vectors, for identification and verification mode and equivalent error rate for verification mode. The results shown that the proposed classifier has a performance better than others previously published, when the leave-one-out method is employed as a test strategy. Best recognition rate of $100 \%$ is achieved with this test methodology.
\end{abstract}

Keywords—Pattern recognition, face recognition, novelty classifier, principal components analysis.

Resumo - Este artigo propõe a utilização do classificado de novidade para reconhecimento de face. Esse classificador é baseado na utilização do filtro de novidade, proposto por Kohonen. O desempenho do novo classificador é comparado com o desempenho do classificador vizinho mais próximo, usando distância euclidiana. A base de dados utilizada para essa comparação foi a base ORL. A informação da face é extraída utilizando PCA e 2DPCA. Os seguintes resultados são apresentados: taxa de reconhecimento versus número de autovetores, no modo de identificação e verificação e taxa de erro equivalente para o modo de verificação. Os resultados obtidos mostraram que o classificador proposto tem um desempenho melhor do que o desempenho do vizinho mais próximo e do que outros classificadores anteriormente publicados usando a mesma base, quando a estratégia Deixa-um-fora (Leave-one-out). A melhor taxa de reconhecimento, $100 \%$, foi obtida com essa metodologia de teste.

Palavras-chave— Reconhecimento de padrões, reconhecimento facial, filtro de novidades, Análise de Componentes Principais.

\section{Introdução}

O reconhecimento facial é um dos tópicos de pesquisa mais atuais na área de reconhecimento de padrões e processamento de imagens. É amplamente usado em medicina, controle de acesso, detecção forense, sistemas de vigilância e monitoração, robótica e interação homem máquina. Sendo assim, possui larga aplicação e valor comercial agregado.

Um sistema de reconhecimento facial geralmente pode ser dividido nas seguintes etapas: aquisição de imagem, pré-processamento, extração de características e classificação. Este trabalho foca na etapa de classificação e não realiza nenhum tipo de préprocessamento nas imagens.

A maioria dos atuais algoritmos de extração de características para reconhecimento de faces é baseado em métodos estatísticos, tais como: PCA (Sirovich e Kirby, 1990; Turk e Pentland, 1991), 2DPCA (Yang et al, 2004), (2D) ${ }^{2}$ PCA (Zhang e Zhou, 2005), LDA (Belhumeur et al, 1997), 2DLDA (Yang et al, 2005), (2D) ${ }^{2}$ LDA (Noushath et al, 2006), entre outros.

Na fase de classificação, destacam-se a aplicação dos seguintes classificadores: Vizinho Mais Próximo associado com a distância euclidiana (Yang et al, 2004; Yang et al, 2005, Zhang e Zhou, 2005,
Noushatha et al, 2006), Redes Neurais (MageshKumar et al, 2011) e Máquina de Vetores de Suporte (Le e Bui, 2011; Oliveira et al, 2011). Perlibakas (2004) comparou 14 distância no reconhecimento facial usando PCA, mostrando os resultados em termos de taxas de reconhecimento rank 1 e erro equivalente. Chan et al (2010) compararam os métodos PCA e LDA no reconhecimento facial nos modos identificação e verificação, mostrando resultados significativos de taxas de reconhecimento rank $1 \mathrm{e}$ erro equivalente. Rouabhia e Tebbikh (2011) propõem a distância matricial ponderada, comparando-a com as distâncias de Yang, Frobenius e distância matricial montada (AMD) usando o método 2DPCA.

Em relação aos resultados publicados na literatura sobre reconhecimento facial, destacamos os seguintes:

A maior taxa de reconhecimento rank 1 atingida por Perlibakas (2004) foi de $85,34 \%$, usando PCA na base ORL em conjunto com a distância do cosseno entre dois vetores, aplicando a transformada whitening descrita em Bishop (1995). O mesmo autor alcançou a menor taxa de erro equivalente, $2,84 \%$ usando a distância de Mahalanobis simplificada.

Chan et al (2010) obteve os melhores resultados na base ORL, usando PCA, a estratégia Leave-oneout e a distância euclidiana. A taxa de reconhecimen- 
to e erro equivalente foram de $91,9 \%$ e $1,15 \%$, respectivamente.

Yang et al (2004) usaram o método Vizinho mais próximo com a métrica da distância euclidiana em seus experimentos. Atingiram uma acurácia de 96\% (estratégia metade treinamento, metade teste) e 98,3\% (Leave-one-out).

Le e Bui (2011) usando 2DPCA e SVM na classificação atingiram $97,3 \%$ de taxa de reconhecimento.

MageshKumar et al (2011) usando PCA e redes neurais na classificação atingiram $98,6 \%$.de taxa de reconhecimento usando estratégia metade treinamento, metade teste.

Rouabhia e Tebbikh (2011) propõem e usam a distância matricial ponderada empregando o método 2DPCA, atingindo a melhor taxa de reconhecimento de $95,5 \%$, utilizando a estratégia metade-metade.

\section{Materiais}

\subsection{Base de faces ORL}

A base de dados ORL (Laboratório de Pesquisa da Olivetti) foi coletada entre 1992 e 1994. Ela contém 10 imagens diferentes de 40 indivíduos (4 mulheres e 36 homens), totalizando 400 imagens de tamanho $92 \times 112$ pixels em escala de cinza. A idade dos indivíduos varia entre 18 à 81 anos, a maioria encontrando-se entre 20 e 35 anos. Para alguns indivíduos, as imagens foram capturadas em diferentes épocas, variando em iluminação, expressões faciais (olhos abertos e fechados, sorrindo ou não) e com detalhes na face (com ou sem óculos), ou seja, não houve uma coleta sistemática. Todas as imagens foram capturadas com um fundo negro homogêneo (Samaria e Harter, 1994; Gross, 2005).

Este trabalho usa a base de faces ORL pelo fato de ser uma das mais usadas pela comunidade científica, por possuir variação na escala e rotação, e por ser possível comparar o desempenho com outros classificadores que a utilizaram.

Neste trabalho é proposto um novo método de classificação no reconhecimento facial, o classificador de novidades, como também, é feita a comparação do desempenho do método proposto com o Vizinho mais próximo associado ao método de extração de características Análise de Componentes Principais (PCA) e 2DPCA. O Classificador de Novidades proposto tem desempenho superior ao Vizinho mais próximo. Uma comparação com os resultados da literatura mostram também o grande potencial do mesmo para reconhecimento facial.

Nessa seção apresentamos o banco de dados de face utilizado nesse trabalho. Na seção de métodos apresentamos o classificador proposto nesse trabalho, bem como o vizinho mais próximo. $\mathrm{Na}$ seção de resultados comparamos os dois classificadores através de curvas de taxa reconhecimento versus o número de autovetores, para os métodos PCA e 2DPCA. São apresentadas também tabelas com os melhores valores de taxa de reconhecimento rank 1 e medidas de erro equivalente para ambos os classificadores. Na seção de discussão comparamos o resultado do classificador proposto nesse trabalho com os resultados de outros trabalhos publicados na literatura.

\section{Métodos}

A metodologia foi desenvolvida com o objetivo de comparar o desempenho dos classificadores Vizinho mais próximo e Classificador de Novidades no reconhecimento de indivíduos através da imagem digital de suas faces.

\subsection{Extração de características}

Sirovich e Kirby (1990) foram os primeiros a usarem PCA eficientemente para representar imagens de faces humanas. Eles argumentaram que qualquer imagem de face poderia ser reconstruída aproximadamente como uma soma ponderada de uma pequena coleção de imagens que definiriam a base de faces (eigenimages), e a média da imagem da face. Dentro deste contexto, Turk e Pentland (1991) apresentaram o método Eigenfaces para reconhecimento facial, o qual já é bastante difundido atualmente. Desde então, PCA tem sido amplamente investigado e tornou-se uma das abordagens de maior sucesso no reconhecimento facial.

Seguindo os passos do PCA, o método de análise de componentes principais bidimensional (2DPCA) foi proposto por Yang et al (2004). Esse método difere do convencional (PCA) pelo fato de usar as matrizes de imagens bidimensionais (2D) diretamente no processo de obtenção da matriz de dispersão, sem a necessidade da transformação da matriz de imagem de um vetor unidimensional (1D). $\mathrm{O}$ algoritmo tem o mesmo objetivo do PCA, que é encontrar vetores de projeção ótimos, que compõem a matriz de projeção. A redução de dimensão é feita na direção horizontal da matriz da imagem. Este método é superior ao PCA em termos de eficiência computacional, pois calcula a matriz de covariância mais rápido. No entanto, o vetor de característica do 2DPCA é maior que o do PCA. Para solucionar este problema é recomendado aplicar PCA após o uso do 2DPCA para redução de dimensionalidade.

\subsection{Vizinho mais próximo}

Após a extração de características usando PCA, ou 2DPCA é comum empregar um classificador Vizinho mais próximo. No presente trabalho, a distância Euclidiana é aplicada, a qual é descrita pela equação (1).

$$
d(a, b)=\sqrt{\sum_{i=1}^{m}\left|a_{i}-b_{i}\right|^{2}}
$$

Na equação (1) $a$ é a imagem de teste e $b$, uma imagem da base de treinamento, ambas projetadas no subespaço gerado pelos métodos de extração de ca- 
racterísticas, onde $m<n$ (n: número total de pixels de uma imagem).

\subsection{Classificador de novidade}

O Classificador de novidades é baseado no filtro de novidades. O conceito de filtro de novidades foi descrito por Kohonen e Oja (1976) baseado no método de ortogonalização clássica de Gram-Schmidt (Kohonen, 1989; Costa e Moura, 1995).

Seja $\left\{x_{1}, x_{2}, x_{3}, \ldots x_{m}\right\} \subset R^{n}$ um conjunto de vetores euclidianos de dimensão $n$ que geram um subespaço de dimensão $m, L \subset R^{n}$, com $m<n$. Considerando o subespaço $L$, um vetor arbitrário $x \in R^{n}$ pode ser decomposto em duas componentes, $\hat{x}$ e $\tilde{x}$, onde $\hat{x} \in L$, e $\tilde{x} \perp L$ é a projeção de $x$ perpendicular a $L$. O vetor $\hat{x} \in L$ representa a componente de $x$ que é "conhecida" pelo subespaço $L$ e pode ser representada como uma combinação linear de $\left\{x_{1}, x_{2}, x_{3}, \ldots x_{m}\right\}$. O vetor $\tilde{x} \perp L$ representa a informação "nova", que é "desconhecida" pelo subespaço $L$ e não pode ser representado como uma combinação linear de $\left\{x_{1}, x_{2}, x_{3}, \ldots x_{m}\right\}$. A Figura 1 ilustra estas duas componentes no espaço $R^{3}$.

Figura 1. Conceito do filtro novidade, mostrando o vetor novidade $\tilde{\mathrm{x}}$ e o vetor projetado $\hat{\mathrm{x}}$

Desta forma, considerando o subespaço $L, \tilde{x}$ é chamado de novidade e o sistema que extrai esta componente de $x$ e a apresenta em sua saída pode ser chamado de Filtro de Novidades.

Para se determinar as componentes $\hat{x}$ e $\tilde{x}$ de um vetor $x$, é usado o processo de ortogonalização de Gram-Schmidt, descrito a seguir. A partir de um conjunto de $m$ vetores independentes $\left\{x_{1}, x_{2}\right.$, $\left.x_{3}, \ldots x_{m}\right\}$, a base $L$, pode-se obter $m$ vetores ortogonais $\left\{v_{1}, v_{2}, v_{3}, \ldots v_{m}\right\}$ através das quações (2) e (3):

$$
v_{1}=x_{1}
$$

$$
v_{k}=x_{k}-\sum_{i=1}^{k-1} \frac{\left(v_{i}, x_{k}\right)}{\left(v_{i}, v_{i}\right)} v_{i}, k=2,3, \ldots, m
$$

$$
\text { Onde } \frac{\left(v_{i}, x_{k}\right)}{\left(v_{i}, v_{i}\right)} v_{i} \text { é a projeção de } x_{k} \text { em } v_{i} \text {. }
$$

Dado um vetor de amostra $x$, sua componente "novidade" é calculada como o $(\mathrm{m}+1)$ passo do processo descrito em (3), ou $\tilde{x}=v_{k+1}$, conforme descrito na equação (4):

$$
\tilde{x}=x-\sum_{i=1}^{m} \frac{\left(v_{i}, x\right)}{\left(v_{i}, v_{i}\right)} v_{i}
$$

A norma do vetor $\tilde{x},\|\tilde{x}\|$, é usada como medida de desigualdade. Quanto menor a norma do vetor $\tilde{x}$, mais próximo ele estará da base $\left\{v_{1}, v_{2}, \ldots v_{m}\right\} \in R^{n}$.

Nesse trabalho propõe-se a utilização do classificador de novidades, que é baseado no filtro de novidade, para reconhecimento de face. A seguir descrevemos um classificador de novidades.

O classificador de novidades utiliza vários filtros de novidades para realização de um único classificador, conforme mostrado no diagrama em blocos da Figura 2. No primeiro nível do diagrama em blocos mostrado constroem-se tantos filtros de novidade quantas imagens de face existirem no banco de faces. No segundo nível do diagrama em blocos mostrado calculam-se as normas dos vetores novidade. Quando uma amostra é aplicada na entrada do classificador, os vetores novidade são então calculados e extraídas as suas normas. No terceiro nível do diagrama em blocos mostrado faz-se uma comparação entre as normas dos vetores de novidade, para se determinar a menor delas. A amostra será classificada como pertencente a face para a qual o filtro de novidade apresentou o menor valor da norma. Assim, se dentre todas as normas a menor delas for $\left|\widetilde{\mathrm{x}_{1}}\right|$, então $\mathrm{s}_{1}=1$, $\mathrm{s}_{\mathrm{i}}=0, \mathrm{i}=2,3 \ldots \mathrm{N}$.

Filtro de

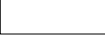


são do vetor em matriz de características, quando necessário.

Os experimentos foram executados nos modos identificação e verificação. Na identificação, analisou-se o impacto do número de components principais (autovetores) no desempenho dos classificadores. $\mathrm{Na}$ verificação, além da análise do impacto do número de components principais no desempenho, extraiu-se as melhores taxas de erro equivalente.

\section{Resultados}

A identificação e verificação facial usando os métodos PCA e 2DPCA associados aos classificadores foram implementados conforme descrito na seção anterior. A Tabela 1 mostra o desempenho dos classificadores no modo identificação. Percebe-se que os classificadores apresentaram taxas de reconhecimento rank 1 mais altas com a estratégia Leave-one-out.

Tabela 1. Maiores taxas de identificação rank 1.

\begin{tabular}{|l|c|c|c|}
\hline \multirow{2}{*}{ Classificador } & Estratégia & Extração & Taxa \\
\hline \multirow{3}{*}{$\begin{array}{l}\text { Classificador } \\
\text { de Novidades }\end{array}$} & $\begin{array}{c}\text { Metade- } \\
\text { metade }\end{array}$ & PCA & $97,5 \%(144)$ \\
\cline { 3 - 4 } & Leave-one- & PCA & $97,5 \%(112 \times 8)$ \\
\cline { 3 - 4 } & out & 2DPCA & $100 \%(25)$ \\
\hline \multirow{3}{*}{$\begin{array}{l}\text { Vizinho mais } \\
\text { próximo }\end{array}$} & $\begin{array}{c}\text { Metade- } \\
\text { metade }\end{array}$ & PCA & $80 \%(112)$ \\
\cline { 3 - 4 } & Leave-one- & PCA & $84,5 \%(112 \times 4)$ \\
\cline { 3 - 4 } & out & PDPCA & $87,5 \%(121)$ \\
\cline { 3 - 4 } & & \multicolumn{2}{|c}{$87,5 \%(112 \times 2)$} \\
\hline
\end{tabular}

As Figura 3 e 4 mostram curvas da taxa de reconhecimento versus o número de autovetores, para o modo de identificação, para ambos os classificadores, utilizando os métodos PCA e 2DPCA, respectivamente.

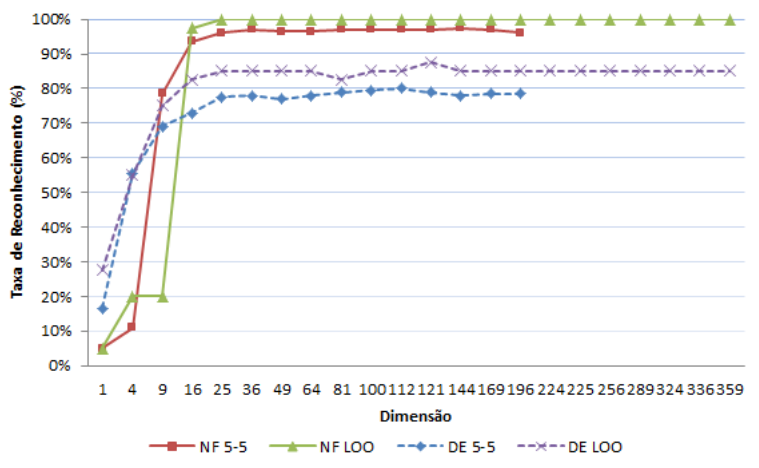

Figura 3. Comparação dos classificadores usando PCA no modo identificação

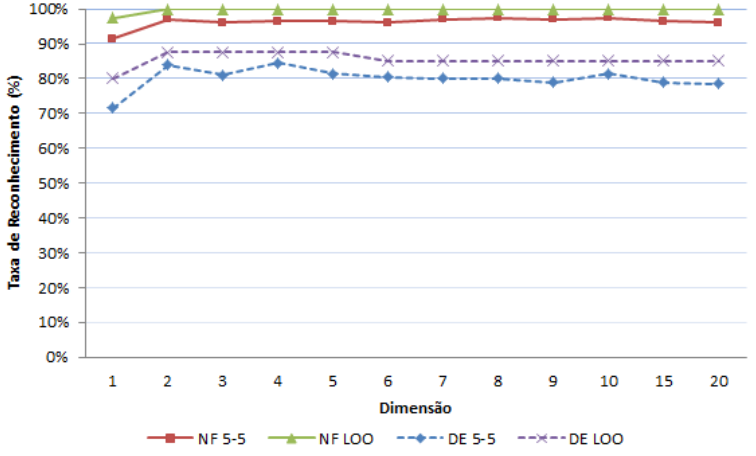

Figura 4. Comparação dos classificadores usando 2DPCA no modo identificação

As Tabela 2 e Tabela 3 mostram o desempenho dos classificadores no modo verificação, apresentando a taxa de verificação e a taxa de erro equivalente (EER). Comparado com o vizinho mais próximo, o classificador de novidades alcançou as menores taxas de erro equivalente no PCA $(3,08 \%$ e $0,03 \%)$ e 2DPCA $(3,00 \%$ e $0 \%)$, nas estratégias metademetade e Leave-one-out, respectivamente.

Tabela 2. Maiores taxas de verificação.

\begin{tabular}{|c|c|c|c|}
\hline Classificador & Estratégia & Extração & Taxa \\
\hline \multirow{4}{*}{$\begin{array}{l}\text { Classificador } \\
\text { de Novidades }\end{array}$} & \multirow{2}{*}{$\begin{array}{l}\text { Metade- } \\
\text { metade }\end{array}$} & PCA & $92 \%(64)$ \\
\hline & & 2DPCA & $93,5 \%(112 \times 2)$ \\
\hline & \multirow{2}{*}{$\begin{array}{l}\text { Leave-one- } \\
\text { out }\end{array}$} & PCA & $100 \%(25)$ \\
\hline & & 2DPCA & $100 \%(112 \times 2)$ \\
\hline \multirow{4}{*}{$\begin{array}{l}\text { Vizinho mais } \\
\text { próximo }\end{array}$} & \multirow{2}{*}{$\begin{array}{l}\text { Metade- } \\
\text { metade }\end{array}$} & PCA & $82,5 \%(25)$ \\
\hline & & 2DPCA & $85 \%(112 \times 4)$ \\
\hline & \multirow{2}{*}{$\begin{array}{l}\text { Leave-one- } \\
\text { out }\end{array}$} & PCA & $90 \%(36)$ \\
\hline & & 2DPCA & $92,5 \%(112 \times 3)$ \\
\hline
\end{tabular}

Tabela 3. Menores taxas de erro equivalente.

\begin{tabular}{|l|c|c|c|}
\hline \multirow{2}{*}{ Classificador } & Estratégia & Extração & EER \\
\hline \multirow{3}{*}{$\begin{array}{l}\text { Classificador } \\
\text { de Novidades }\end{array}$} & Metade-metade & PCA & $3,08 \%$ \\
\cline { 3 - 4 } & Leave-one-out & PDPCA & $3,00 \%$ \\
\cline { 3 - 4 } & & PCA & $0,03 \%$ \\
\hline \multirow{2}{*}{$\begin{array}{l}\text { Vizinho mais } \\
\text { próximo }\end{array}$} & Metade-metade & PCA & $0,00 \%$ \\
\cline { 3 - 4 } & Leave-one-out & 2DPCA & $5,10 \%$ \\
\cline { 3 - 4 } & & PCA & $3,08 \%$ \\
\cline { 3 - 4 } & & 2DPCA & $2,92 \%$ \\
\hline
\end{tabular}

As Figura 5 e 6 mostram curvas da taxa de reconhecimento e taxa de erro equivalente, respectivamente, versus o número de autovetores, para o modo verificação, para ambos os classificadores, utilizando o método PCA.

As Figura 7 e 8 mostram curvas da taxa de reconhecimento e taxa de erro equivalente, respectivamente, versus o número de autovetores, para o modo verificação, para ambos os classificadores, utilizando o método 2DPCA. 


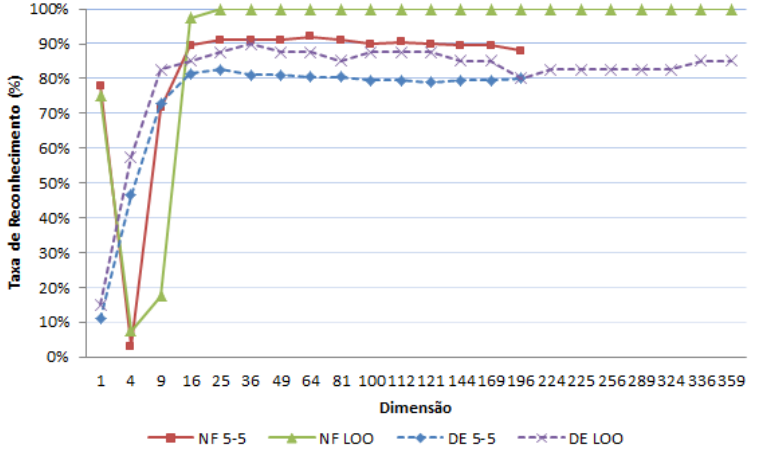

Figura 5. Comparação dos classificadores usando PCA no modo verificação

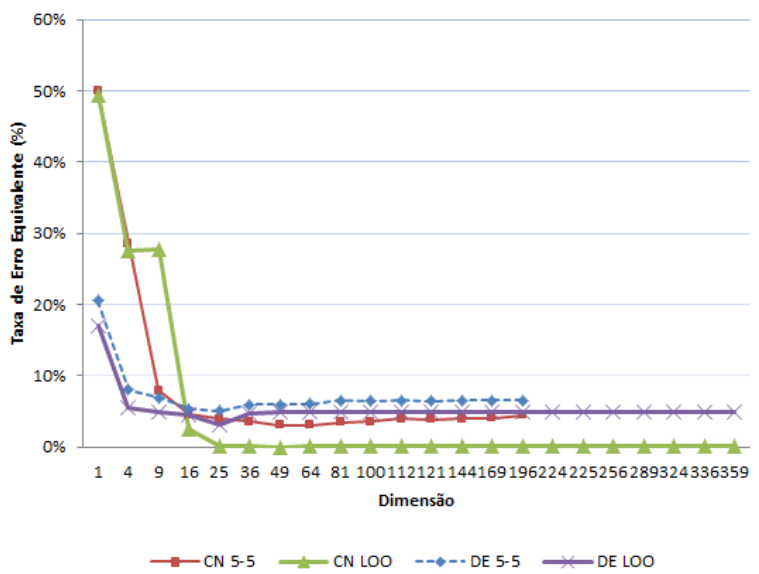

Figura 6. Taxa de erro equivalente usando o método PCA

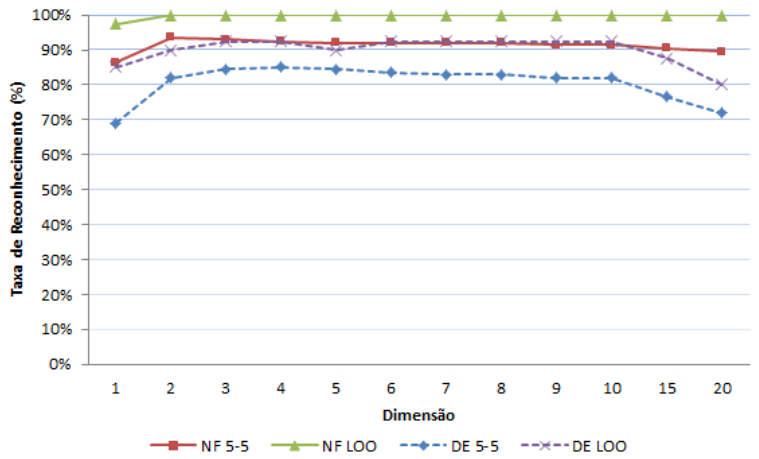

Figura 7. Comparação dos classificadores usando 2DPCA no modo verificação

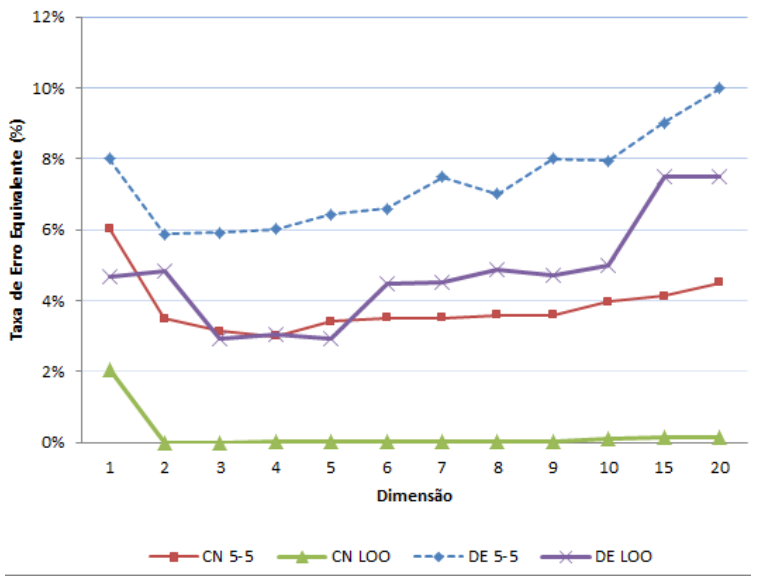

Figura 8. Taxa de erro equivalente usando o método 2DPCA
Embora a taxa de identificação rank1 usando PCA seja muito alta quando comparada em classificado do vizinho mais próximo, alguns erros foram cometidos. Na Figura 9 mostramos um erro de identificação cometido. Na Figura 9(a) mostramos um grupo de 5 imagens de um indivíduo A utilizadas para compor a base do filtro de novidades desse indivíduo. Na Figura 9(b) mostramos outro grupo de 5 imagens de um indivíduo B utilizadas para compor a base do filtro de novidades desse indivíduo. Na Figura 9(c) mostramos uma sexta imagem do indivíduo A que foi identificada pelo classificador de novidade como sendo do indivíduo B. O valor da novidade da imagem da Figura 9(c) em relação a base mostrada na Figura 9(a) foi de 2111,82, enquanto que o valor da novidade da mesma imagem em relação à base mostrada na Figura 9(c) foi de 2024,23. Aparentemente, não existe nenhuma razão para que essa identificação tenha sido feito erroneamente. Assim sendo, mais estudos precisam ser realizados para que se chegue a uma compreensão mais aprofundada do modo como opera o filtro de novidades.

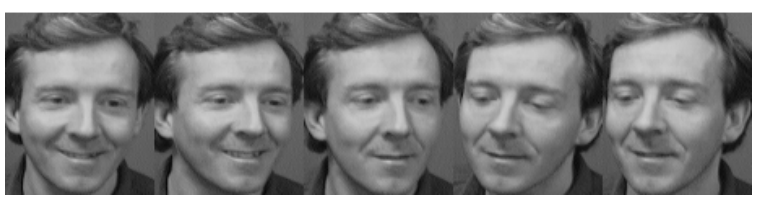

(a)

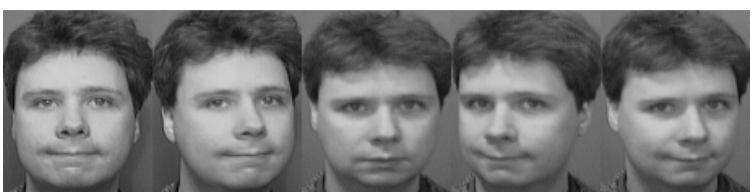

(b)

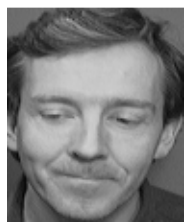

(c)

Figura 9. (a) 5 imagens que compõem a base de um indivíduo A; (b) 5 imagens que compõem a base de um indivíduo B; (c) Uma sexta imagem do indivíduo A que foi identificada pelo classificador de novidade como sendo do indivíduo B.

\section{Discussão}

Através dos resultados apresentados na seção anterior verificamos que:

1. Ao comparar os resultados apresentados nesse trabalho em relação ao Vizinho mais próximo e ao Classificador de Novidades, verificamos que esse último apresentou resultados bem superiores, tanto no que diz respeito à taxa de reconhecimento como à taxa de erro equivalente; 
2. Ao comparar os resultados apresentados nesse trabalho para o Classificador de Novidades com os resultados da literatura anteriormente expostos, verificamos que o mesmo apresenta os melhores resultados com a estratégia Leave-one-out. Com respeito a estratégia metade-metade o Classificador de Novidades atingiu resultados inferiores aos publicados por MageshKumar et al (2011), porém superiores ao resultados apresentado por Rouabhia e Tebbikh (2011). Alguns autores não especificam a estratégia adotada, o que inviabiliza uma comparação rigorosa.

\section{Conclusão}

Foi proposto um novo método para classificação no reconhecimento facial: o Classificador de Novidades. Para testar e avaliar o desempenho do classificador proposto, o mesmo foi comparado o Vizinho mais próximo e uma série de experimentos envolvendo identificação e verificação biométrica da face foram executados usando a base de faces ORL. Os resultados mostraram que o classificador de novidades é eficaz no reconhecimento facial usando as bem conhecidas técnicas de extração de características: PCA e 2DPCA. Tendo em vista consolidar a utilização do classificador ora proposto, planeja-se como trabalhos futuros: o uso das estratégias (2D) ${ }^{2} \mathrm{PCA}, \mathrm{LDA}$, 2DLDA e (2D) ${ }^{2}$ LDA; a utilização de outras bases de face e uma comparação com outras distâncias, tais como distância de Mahalanobis, do cosseno, entre outras.

\section{Agradecimentos}

A produção deste trabalho teve o suporte do CNPq, processo 470972/2011-4, FAPEAM e Instituto Nokia de Tecnologia.

\section{Referências Bibliográficas}

Kirby, M. and Sirovich, L. (1990). Application of the KL Procedure for the Characterization of Human Faces. IEEE Trans. Pattern Analysis and Machine Intelligence, vol. 12, no. 1; pp. 103-108. DOI: $10.1109 / 34.41390$

Turk, M. and Pentland, A. (1991). Eigenfaces for Recognition. J. Cognitive Neuroscience, vol. 3, no. 1, pp. 71-86. DOI: 10.1162/jocn.1991.3.1.71

Yang, J.; Zhang, D.; Frangi, A. and Yang, J. (2004). Two-Dimensional PCA: A New Approach to Appearance-Based Face Representation and Recognition. IEEE Trans. Pattern Analysis and
Machine Intelligence, vol. 26, no. 1, pp. 131137. DOI: 10.1109/TPAMI.2004.1261097

Zhang, D.; Zhou, Z. (2005). (2D)²PCA: Twodirectional two-dimensional PCA for efficient face representation and recognition. Neurocomputing, Vol. 69, P. 224-231. DOI: 10.1016/ j.neucom.2005.06.004

Noushath, S.; Kumat, G. H.; Shivakumara, P. (2006). (2D) ${ }^{2}$ LDA: An efficient approach for face recognition. Pattern Recognition, Vol. 39, P. 1396-1400.

Belhumeur, P.; Hespanha, J.; Kriengman, D. (1997). Eigenfaces vs. Fisherfaces: recognition using class specific linear projection, IEEE Trans. Pattern Anal. Machine Intell. 19 (7) 711-720.

Yang, J.; Zhang, D.; Yong, X.; Yang, J. (2005). Two-dimensional discriminant transform for face recognition. Pattern Recognition Society, Vol. 38, P. 1125-1129.

Oliveira, L.; Koerich, A.L.; Mansano, M.; Britto, A.S. (2011). 2D Principal Component Analysis for Face and Facial-Expression Recognition. Computing in Science \& Engineering, vol.13, no.3, pp.9-13.

MageshKumar, C.; Thiyagarajan, R.; Natarajan, S.P.; Arulselvi, S.; Sainarayanan, G. (2011). Gabor features and LDA based face recognition with ANN classifier. Emerging Trends in Electrical and Computer Technology (ICETECT), pp.831836, 23-24.

Perlibakas, V. (2004). Distance measures for PCAbased face recognition. Pattern Recogn. Lett. 25, 6, 711-724. DOI: 10.1016/j.patrec.2004.01.011

Bishop, C.M. (1995). Neural Networks for Pattern Recognition. Clarendon Press, Oxford. p. 504.

Chan, L.; Salleh, S; Ting, C. (2010). Face Biometrics Based on Principal Component Analysis and Linear Discriminant Analysis. Journal of Computer Science;2010, Vol. 6 Issue 7, p691.

Rouabhia, C.; Tebbikh, H. (2011). Efficient face recognition based on weighted matrix distance metrics and 2DPCA algorithm. Archives of Control Sciences, Vol. 21 Issue 2, p207.

Kohonen, T., Oja, E.. (1976). Fast adaptive formation of orthogonalizing filters and associative memory in recurrent networks of neuron-like elements. Biological Cybernetics (BIOL CYBERN), vol. 25, no. 2, pp. 85-95. SpringerVerlag, Berlin.

Kohonen, T. (1989). Self-Organization and Associative Memory, ed. 3rd. Springer-Verlag, BerlinHeidelberg, New York-Tokyo.

Costa, M.; Moura, L. (1995). Automatic assessment of scintmammographic images using a novelty filter. Proceedings of the 19th Annual Symposium on Computer Applications in Medical Care, pp. 537-41. 